JOURNAL OF

SYNCHROTRON

RADIATION

ISSN 1600-5775

Received 27 April 2018

Accepted 13 July 2018

Edited by P. A. Pianetta, SLAC National Accelerator Laboratory, USA

Keywords: phase-contrast breast CT; synchrotron radiation; breast cancer imaging.

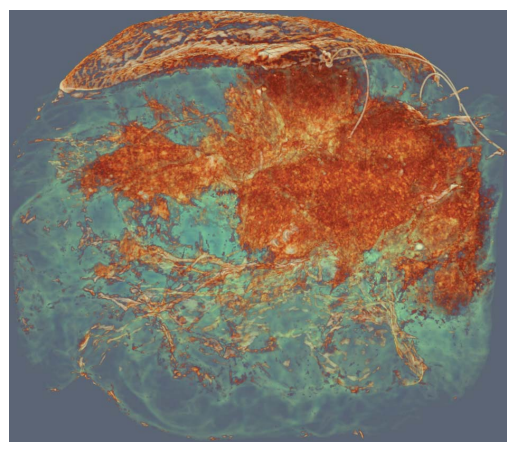

C 2018 International Union of Crystallography

\section{Advantages of breast cancer visualization and characterization using synchrotron radiation phase-contrast tomography}

\author{
Serena Pacilè, ${ }^{\mathrm{a}, \mathrm{b} *}$ Patrycja Baran, ${ }^{\mathrm{c}}$ Christian Dullin, ${ }^{\mathrm{a}, \mathrm{d}, \mathrm{e}}$ Matthew Dimmock, ${ }^{\mathrm{f}}$ \\ Darren Lockie, ${ }^{\mathrm{g}}$ Jeannine Missbach-Guntner, ${ }^{\text {d }}$ Harry Quiney, ${ }^{\mathrm{c}}$ \\ Mikkaela McCormack, ${ }^{\text {h }}$ Sheridan Mayo, ${ }^{i}$ Darren Thompson, ${ }^{,}$Yakov Nesterets, ${ }^{j}$ \\ Chris Hall, ${ }^{k}$ Konstantin Pavlov, ${ }^{\mathrm{j}, \mathrm{l}, \mathrm{m}}$ Zdenka Prodanovic, ${ }^{\mathrm{n}}$ Maura Tonutti, ${ }^{\circ}$ \\ Agostino Accardo, ${ }^{\mathrm{b}}$ Jane Fox, ${ }^{\mathrm{n}, \mathrm{p}}$ Seyedamir Tavakoli Taba, ${ }^{\mathrm{q}}$ Sarah Lewis, ${ }^{\mathrm{q}}$ \\ Patrick Brennan, ${ }^{\mathrm{q}}$ Daniel Hausermann, ${ }^{\mathrm{k}}$ Giuliana Tromba ${ }^{\mathrm{a}}$ and Tim Gureyev ${ }^{\mathrm{c}, \mathrm{i}, \mathrm{j}, \mathrm{m}}$
}

aElettra Sincrotrone Trieste, Basovizza, Trieste 34149, Italy, ${ }^{\mathbf{b}}$ Department of Engineering and Architecture, University of Trieste, Trieste, Italy, ${ }^{\mathbf{C} A R C}$ Centre of Excellence in Advanced Molecular Imaging, School of Physics, University of Melbourne, Parkville, Australia, ${ }^{\mathbf{d}}$ Institute for Diagnostic and Interventional Radiology, University Medical Center Göttingen, Göttingen, Germany, ${ }^{\mathbf{e}}$ Translational Molecular Imaging, Max-Planck-Institute for Experimental Medicine, Göttingen, Germany, 'Department of Medical Imaging and Radiation Sciences, Monash University, Clayton, Australia, g Maroondah BreastScreen, Ringwood East 3135, Australia, 'horevitch Pathology, Heidelberg 3084, Australia, 'Commonwealth Scientific and Industrial Research Organisation, Clayton, Australia, 'S School of Science and Technology, University of New England, Armidale, Australia, ' Australian Synchrotron, Clayton, Australia, 'School of Physical and

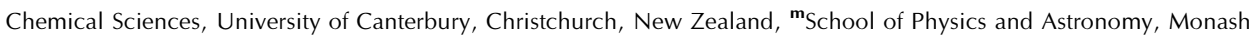
University, Clayton, Australia, ${ }^{\mathbf{n}}$ Monash Health, Clayton, Australia, ${ }^{\mathbf{o}}$ Department of Radiology, Academic Hospital of Trieste, Trieste, Italy, ${ }^{\mathbf{p}}$ Nursing and Health Sciences, Monash University, Clayton, Australia, and ${ }^{\mathbf{q}}$ Medical Image Optimisation and Perception Group, Faculty of Health Sciences, University of Sydney, Sydney, Australia.

*Correspondence e-mail: serena.pacile@elettra.eu

The aim of this study was to highlight the advantages that propagation-based phase-contrast computed tomography (PB-CT) with synchrotron radiation can provide in breast cancer diagnostics. For the first time, a fresh and intact mastectomy sample from a 60 year old patient was scanned on the IMBL beamline at the Australian Synchrotron in PB-CT mode and reconstructed. The clinical picture was described and characterized by an experienced breast radiologist, who underlined the advantages of providing diagnosis on a PB-CT volume rather than conventional two-dimensional modalities. Subsequently, the image quality was assessed by 11 breast radiologists and medical imaging experts using a radiological scoring system. The results indicate that, with the radiation dose delivered to the sample being equal, the accuracy of a diagnosis made on PB-CT images is significantly higher than one using conventional techniques.

\section{Introduction}

The most important factor to increase the survival rate in breast cancer is early detection of breast cancer lesions (Siegel et al., 2018). The standard method for diagnosis of breast cancer is mammography, a method with well documented limitations (Elmore et al., 2005; Joy et al., 2005; HeywangKöbrunner et al., 2011). Mammography is a two-dimensional projection technique which, despite its excellent spatial resolution, can limit the detection and visualization of cancer, as soft-tissue tumour masses and calcifications can be hidden behind overlapping normal tissue. The poor contrast between healthy and cancerous tissue, and the need to discriminate minute and subtle details in a lesion within the breast, require an imaging platform that has adequate spatial and contrast resolution. Furthermore, breast density and tissue distribution vary among women, increasing the complexity of image interpretation, particularly for women with dense breasts 
(Sprague et al., 2017). Recent technical developments have led to the introduction of digital breast tomosynthesis (DBT) to clinical routine (Ciatto et al., 2013; Alakhras et al., 2013; Michell et al., 2012). In this technique, multiple angled projection images are produced, allowing visual review of relatively thin breast sections and thus offering the potential to unmask cancers that would have been obscured in mammography through superimposition by normal tissue located above and/or below the lesion. Nevertheless, the small difference in X-ray attenuation between the glandular and tumour tissues remains a problem for this imaging method.

In the light of these considerations, there is a strong need for improvements in the currently available methods for breast imaging (Kidane et al., 1999; Zhao et al., 2012). A tomographic approach in combination with phase-contrast techniques could overcome these problems and, at the same time, provide a three-dimensional visualization of the breast, allowing the depiction of three-dimensional morphology and lesion distribution. This could lead to an improvement in sensitivity, as it would allow the detection of both smaller lesions and cancers not visible by conventional mammography, and in screening specificity as well, as it would allow a better visualization of the margin of a mass or its asymmetry, leading to a more detailed characterization of the lesions and potentially reducing the number of missed cancers and falsepositive results.

In recent years, different approaches for phase-based X-ray imaging methods have been explored and applied to breast imaging, demonstrating important improvements in breast cancer diagnosis (Pagot et al., 2005; Sztrókay et al., 2012; Diemoz et al., 2012; Keyriläinen et al., 2011; Coan et al., 2013; Bravin et al., 2013; Tromba et al., 2016). With the aim of translating the phase-contrast computed tomography (PB-CT) technique into clinical practice, we have carried out a series of work on feasibility studies and optimization of the main parameters (Pacilè et al., 2015; Baran et al., 2017; Nesterets et al., 2015; Longo et al., 2016). In this paper, starting from the results obtained in these previous studies, we used an optimized acquisition protocol to scan an entire excised breast, shortening the distance between the previous feasibility works and the in vivo application of the PB-CT technique. Furthermore, we provide an example of the improvement in lesion detection using synchrotron radiation phase-contrast breast $\mathrm{CT}$ in comparison with conventional methods used in current clinical routine (digital mammography, ultrasound and DBT).

\section{Materials and methods}

\subsection{Sample description}

A fresh and intact mastectomy sample from a 60 year old woman, delivered directly from Monash Health, a local operating centre, was used in this study. The excised specimen (hereafter referred to as Right Breast) contained an invasive papillary carcinoma with focal skin invasion, composed of multiple nodules through the scar tissue as identified by an experienced pathologist. The presence of the scar tissue is due to the fact that the patient had previously undergone surgery and radiation therapy for early breast cancer, and hence this was a recurrent breast cancer in a previously conserved breast. This research has been carried out in accordance with the Australian National Statement on Ethical Conduct in Human Research. The Ethics Approval was granted by the Monash University Human Research Ethics Committee on 19 August 2015 (project No. CF15/3138-2015001340).

\subsection{Imaging technique}

The experiment was conducted on the Imaging and Medical Beamline (IMBL) at the Australian Synchrotron (Clayton, Melbourne). CT scans were collected, in propagation-based imaging mode (PB-CT) (Rigon, 2014; Diemoz et al., 2012), at a sample-to-detector distance of $5.71 \mathrm{~m}$ using monochromatic $\mathrm{X}$-rays with an energy of $32 \mathrm{keV}$. These optimized conditions were selected based on the results of our previous work (Baran et al., 2017; Pacilè et al., 2015) in which the effect of different experimental parameters on the image quality was evaluated. For each scan, we acquired 1800 projections equally distributed over $180^{\circ}$, with a detector exposure time of $0.05 \mathrm{~s}$ per projection. The dose delivered to the samples was about $4 \mathrm{mGy}$, calculated using a numerical phantom that simulates a breast composed of $50 \mathrm{wt} \%$ gland tissue and $50 \mathrm{wt} \%$ adipose tissue surrounded by adipose tissue (simulating the skin layer), as done by Baran et al. (2017). This dose level is comparable with a standard mammogram and tomosynthesis examination. The detector was a Hamamatsu CMOS flat-panel sensor C9252DK-14, which contains a CsI scintillator deposited directly on a two-dimensional photodiode array. The detector was used in the partial scan mode with a pixel size of $100 \mu \mathrm{m} \times$ $100 \mu \mathrm{m}$.

\subsection{Data processing and analysis}

For CT data processing, the $X$-TRACT analysis software (Gureyev et al., 2011) was used, which includes data preprocessing, phase retrieval and CT reconstruction. The preprocessing of projection images contained dark-current and flatfield correction and the application of a ring-removal filter. Phase retrieval was performed using the TIE-Hom algorithm (Paganin et al., 2002), with a ratio between the refracting decrement $(\delta)$ and the absorption component $(\beta)$ of the refractive index $n$ (Paganin, 2013) close to the theoretical value for glandular tissue in adipose tissue (http://www. ts-imaging.net). For $\mathrm{CT}$ reconstruction, the iterative filtered back-projection (iFBP) algorithm also implemented in $X$-TRACT was used. The diagnostic accuracy was evaluated in comparison with clinical digital mammography, medical ultrasound and DBT, which were performed prior to surgical intervention, using the reconstructed CT data sets and threedimensional renderings. Lastly, to investigate further the contribution that phase-contrast retrieval specifically contributes to better image quality, 11 assessors, including six radiologists and five medical imaging experts, performed a systematic radiological assessment between a reference image produced using the same source of X-rays but without any 
phase-contrast retrieval, and images produced when the phase-contrast component of the image was included. The image-quality criteria for the evaluated images were:

(i) Soft-tissue contrast (degree of contrast of various softtissue regions).

(ii) Edge sharpness (clarity of definition of edges and outlines of structures).

(iii) Soft-tissue interfaces (clarity of visualization of interfaces between different soft-tissue elements).

(iv) Spiculations (sharpness of reproduction of spiculations).

(v) Image noise (distractiveness of noise in the image).

(vi) Calcification visibility (ability to visualize presence of micro-calcifications).

\section{Results and discussion}

\subsection{Description and characterization of the clinical picture}

At present, the advantages of breast PB-CT have only been demonstrated in fixed and partial breast specimens (quadrantectomies or slices) that do not replicate the real clinical situation (Baran et al., 2017; Pacilè et al., 2015; Longo et al., 2016). Here, for the first time, we present results obtained in a fresh and intact mastectomy sample which give a better evaluation of the potential clinical impact of PB-CT. To illustrate further the advantages that PB-CT could bring to the diagnostic process, it is necessary to start from diagnoses made on the basis of mammography, tomosynthesis and ultrasound examination, and then highlight the differences.

The mammography examination (Fig. 1) of the patient shows an asymmetry of the glandular tissue between the two breasts, a condition that is often caused by a lobular carcinoma. The glandular tissue of the Right Breast has an architectural distortion and involves micro-calcifications (red circles in Fig. 1, left), and furthermore the images are compatible with a skin invasion (yellow arrows in Fig. 1, right),

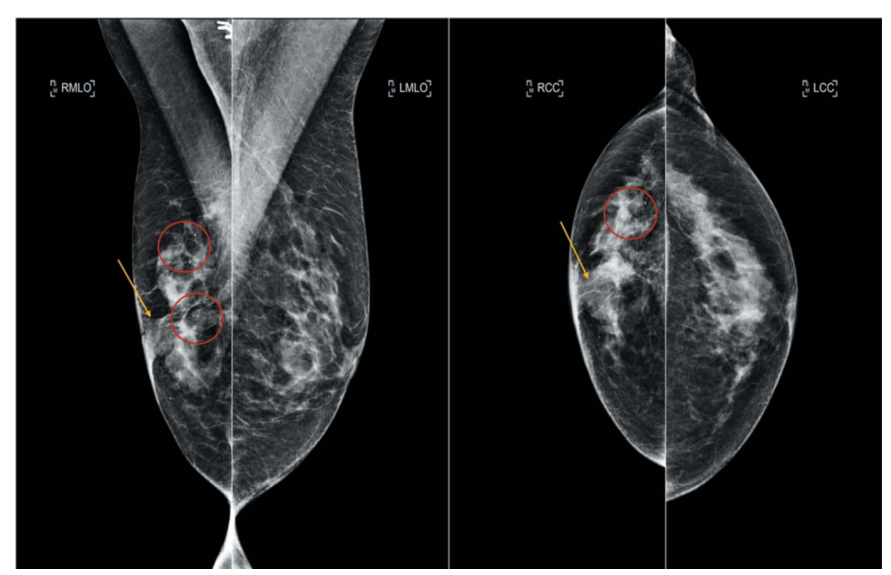

Figure 1

Digital mammography of the 60 year old patient from which the Right Breast sample was excised. On the left-hand side are medio-lateral oblique views of both breasts, and on the right-hand side are craniocaudal views. Red circles indicate areas containing micro-calcifications. Yellow arrows mark the suspected skin invasion. as the portion of the skin near the nipple is thicker and denser than the remaining breast skin. However, from the mammographic images it is not possible to characterize the margins of the mass. On the DBT images (Fig. 2), the opacities show clear spiculation, allowing the radiologist to identify the malignant nature of the lesion.

From the ultrasound examination, multiple lesions are visible: a suspicious solid lesion $6 \mathrm{~cm}$ from the nipple (Fig. $3 a$ ), a hypoechoic lesion in the upper quadrant with a diameter of $1.82 \mathrm{~cm}$ (white markers in Fig. $3 b$ ) containing calcifications at $3 \mathrm{~cm}$ from the nipple (Fig. $3 b$ ), and neoplastic tissue with calcifications inside the ducts in the retroareolar region (Figs. $3 c$ and $3 d$ ). This clinical picture is compatible with a multicentric tumour with in situ component and lymph nodes.

The reconstructed three-dimensional images obtained from the synchrotron radiation $\mathrm{CT}$ scans can be visualized in three orthogonal planes (transverse, sagittal and coronal; Fig. 4) and with three-dimensional rendering (Fig. 6). The transverse and sagittal breast CT views correspond to the mammographic cranio-caudal and mediolateral views. The dimensions of the lesion are measurable $(6.5 \mathrm{~cm})$ and its malignant characteristics can be clearly assessed. In contrast with mammography, in which it is not easy to understand whether a region is malignant or healthy, here the parenchymal distortion and spiculations are clearly delineated. In addition, the invasion of the tumour into the skin, a diagnosis that can be suggested on the mammography and tomosynthesis images, is clearly noticeable in the PB-CT images from the solid tissue expanding toward the areola (sagittal view of the sample in Fig. 4).

The histological examination confirmed the presence of a field of scarring in the upper quadrants, with multifocal invasive papillary carcinoma and associated high nuclear grade DCIS (ductal carcinoma in situ) through this region. Besides these ductal lesions, a prominent feature of the tumour mass is the augmented accumulation of dense fibrotic tissue with

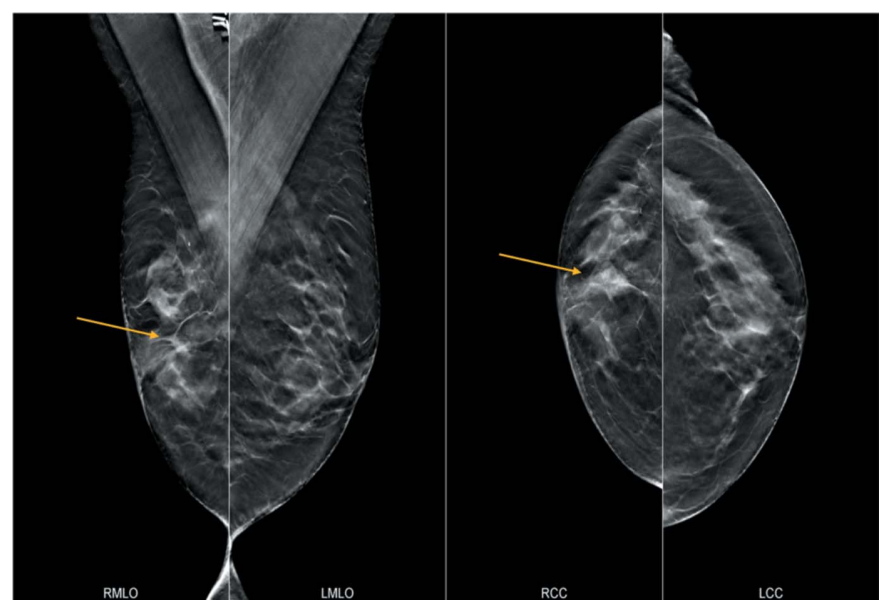

Figure 2

Tomosynthesis of the patient before surgical mastectomy. On the lefthand side are medio-lateral views of both breasts (corresponding to the sagittal plane), and on the right-hand side are cranio-caudal views (corresponding to the transverse plane). Yellow arrows indicate the spiculations expanding from the cancerous area. 


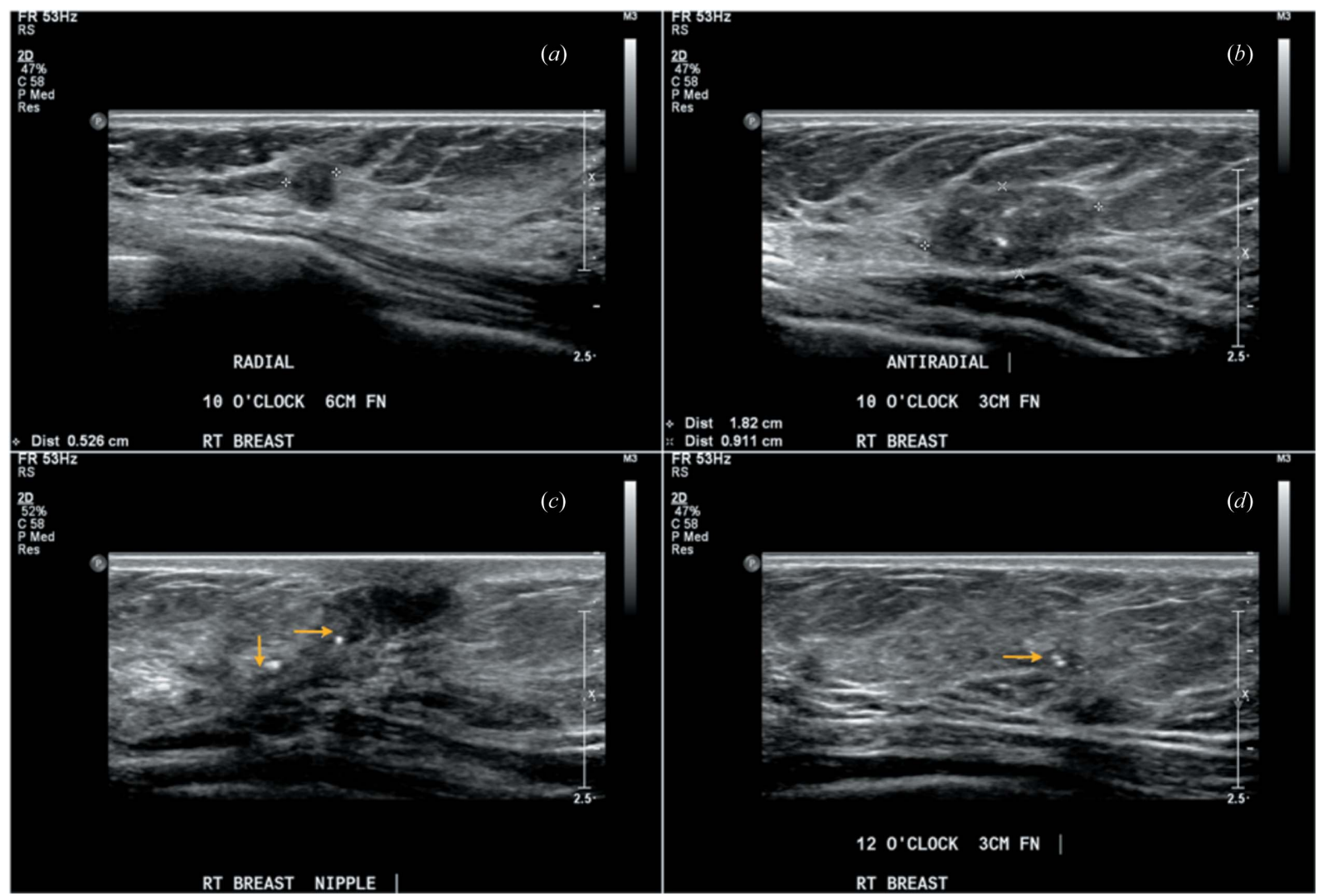

Figure 3

Ultrasound examination of Right Breast. (a) The solid lesion with a diameter of $0.526 \mathrm{~cm}$, located $6 \mathrm{~cm}$ away from the nipple, delimited by white markers.

(b) The oval delimited by the white markers is the hypoechoic lesion $3 \mathrm{~cm}$ away from the nipple, including microcalcification (brighter spots). (c) and $(d)$ The neoplastic tissue with calcifications (indicated by yellow arrows) inside the ducts in the retro-areolar region.
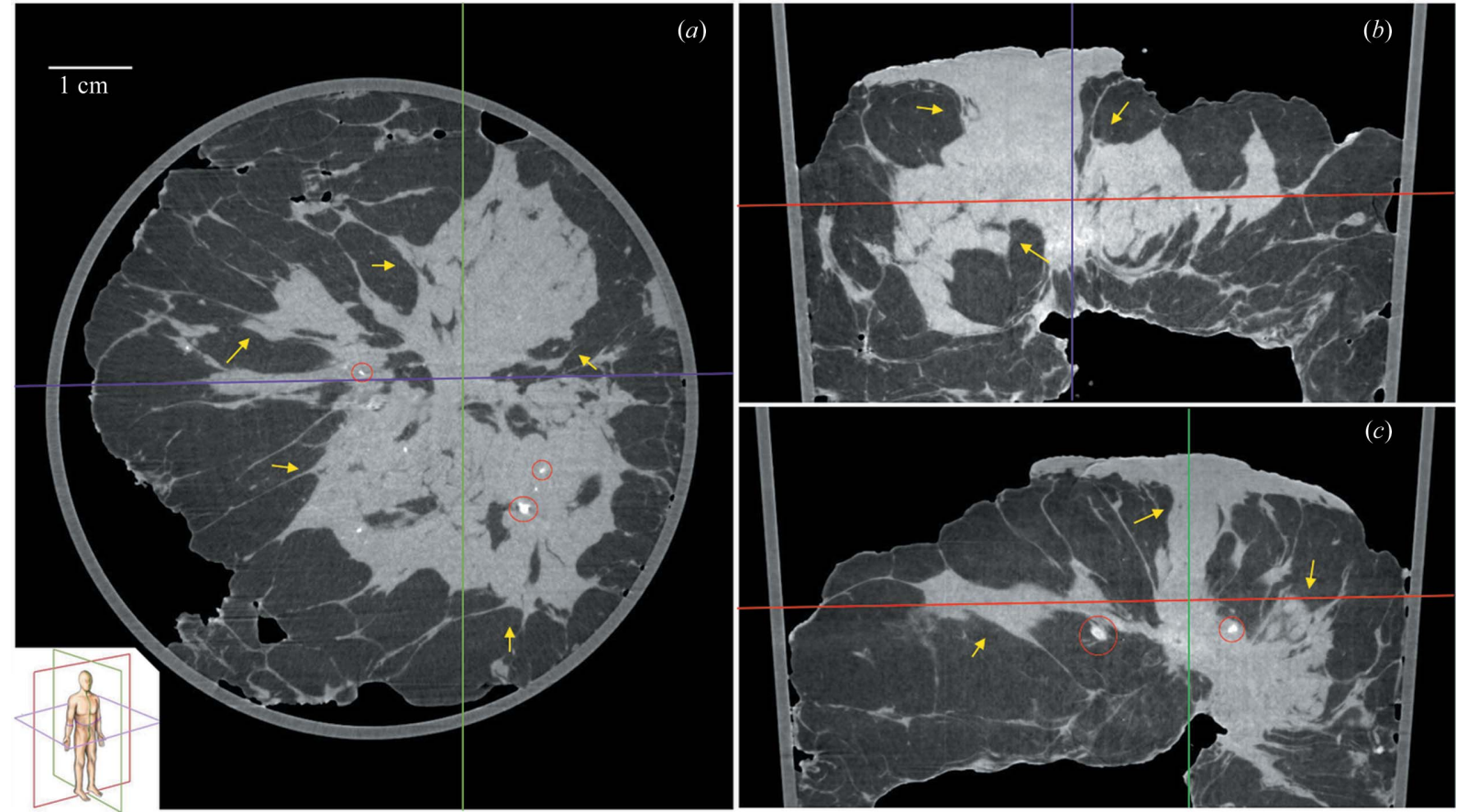

Figure 4

Tomographic reconstruction of Right Breast with a dense accumulation of tumorous fibrotic tissue (yellow arrows) and the presence of microcalcifications (circles). On the left in panel $(a)$ is the conventional view (plane parallel to the X-ray beam). On the right are the two related orthogonal views, $(b)$ in the sagittal plane and $(c)$ in the transverse plane. Both $(b)$ and $(c)$ show a portion of the skin including the nipple in their upper part. Coloured lines indicate the positions of the corresponding orthogonal sections, with the red line representing the coronal plane, green the sagittal plane and violet the transverse plane. The data set was acquired at $5.71 \mathrm{~m}$ and $32 \mathrm{keV}$, using the iterative FBP reconstruction method and preprocessing the data with the TIE-Hom phase-retrieval algorithm. 

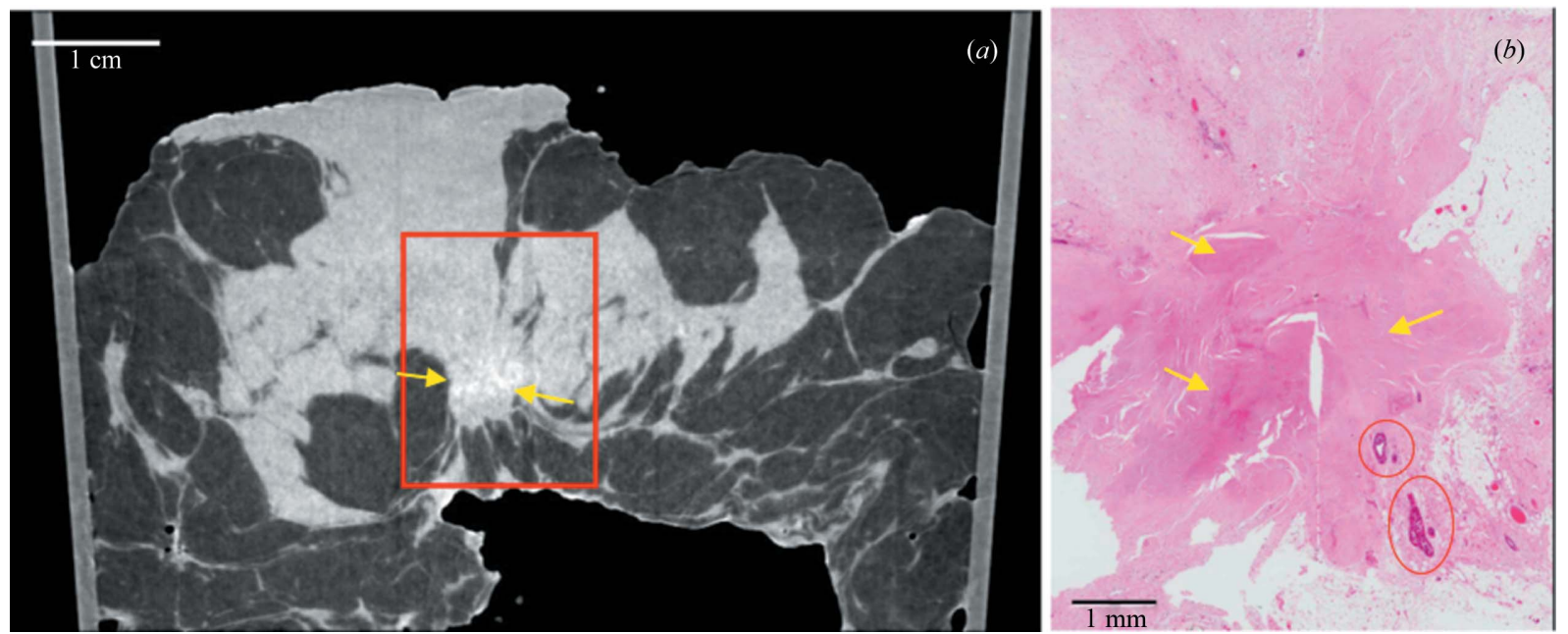

Figure 5

The correspondence between $(a)$ a CT sagittal image and $(b)$ a histology image. The histological examination shows the diffuse infiltrating tumour mass, consisting mainly of a dense accumulation of fibrotic matrix-rich tissue (arrows). This scar-like tumorous mass extensively replaces the parenchymal compartment of the breast. The few peripherally located glandular ducts display an augmented epithelial hyperplasia (circles). These histopathological findings are in line with the high radio opacity of the dense tissue mass detected within the synchrotron data set [panel (a), arrows].

extensive displacement of breast parenchyma. The tumour spans $65 \mathrm{~mm}$, with 17 foci of invasive carcinoma up to $16 \mathrm{~mm}$ and two additional skin nodules of $3 \mathrm{~mm}$ each without skin ulceration, clear of all planes of resection. In general, the tumour type is difficult to define, as in some areas the tumour cells form small tubules resembling a carcinoma of no special type, and these areas are blended with areas where the tubules become larger with a micropapillary architecture resembling micropapillary DCIS. As these foci enlarge further, they develop true papillae with fibrovascular stromal cores. For this

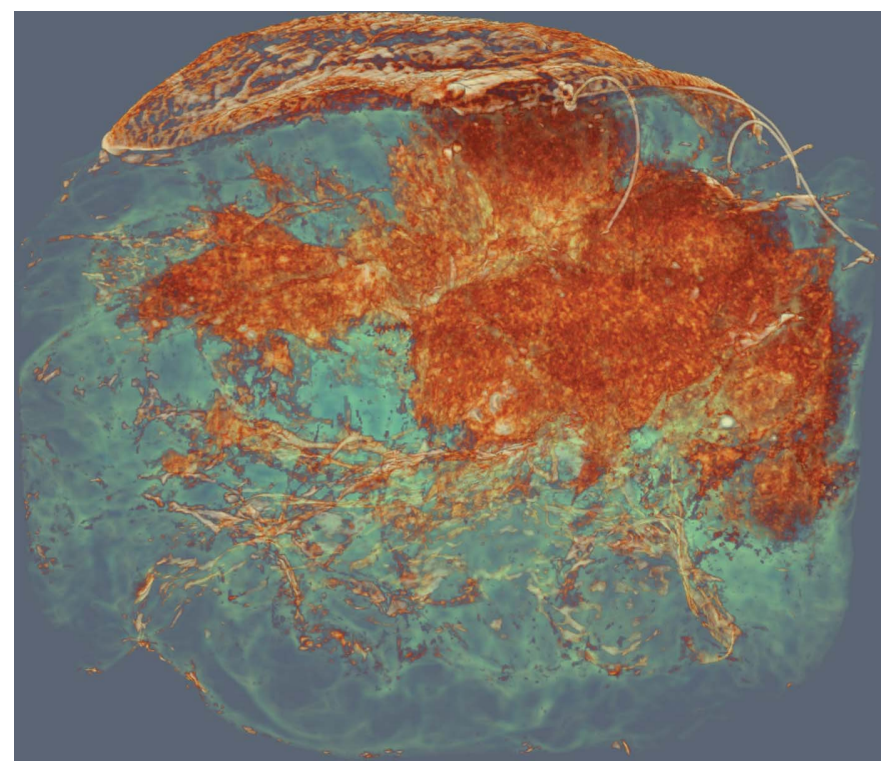

Figure 6

Three-dimensional rendering of Right Breast. Colours are associated with an increasing level of density from green (lowest) to red (highest and associated with cancerous tissue). In the upper part of the sample there is a portion of skin including the nipple. The long suture wire is placed approximately at the midpoint of the lateral edge (right side of the body) while the short one indicates the midpoint of the superior edge (toward the head end of the body). reason, the tumour has been classified as an invasive papillary carcinoma. For some of these histological images, the corresponding X-ray PB-CT images were identified. An example is shown in Fig. 5.

Another major advantage of the tomographic modality is the possibility of obtaining a three-dimensional rendering of the entire examined volume. The presented renderings were obtained using the Avizo software, a proprietary software package for three-dimensional data analysis produced by Thermo Scientific. Fig. 6 shows a depiction of the Right Breast based on the different density values: the yellow and red parts are the densest ones (in increasing order of density from red to yellow) and are associated with skin (on the top of the sample) and cancerous tissue. The green part is less dense and is associated with adipose tissue; it is almost transparent, with just the greenish wisps to show the material which is not fat but not quite as high density as the bulk tumour region. In addition to $\mathrm{CT}$ visualization, the three-dimensional rendering, thanks to its depth perception, allows a global analysis of the mass with a better characterization of shape, margins, extent of spiculations etc. This could provide a strong rationale for assessing tumour features, for example, throughout the course of chemotherapy, or to give a precise position map in the case of a multifocal lesion in pre-surgical planning.

\subsection{Radiological assessment}

The assessors used a five-point scoring scale (from -2 to 2) to rate the fulfilment of each criterion in the PB-CT images in comparison with the reference images approximating conventional absorption-based CT images (the reference image was obtained using the following conditions: $0.16 \mathrm{~m}$ distance, $32 \mathrm{keV}$ X-ray energy, using the SIRT1000 reconstruction method and no phase retrieval). In total, 198 observations (related to three images, six quality criteria and 11 assessors) were undertaken. The intraclass correlation 

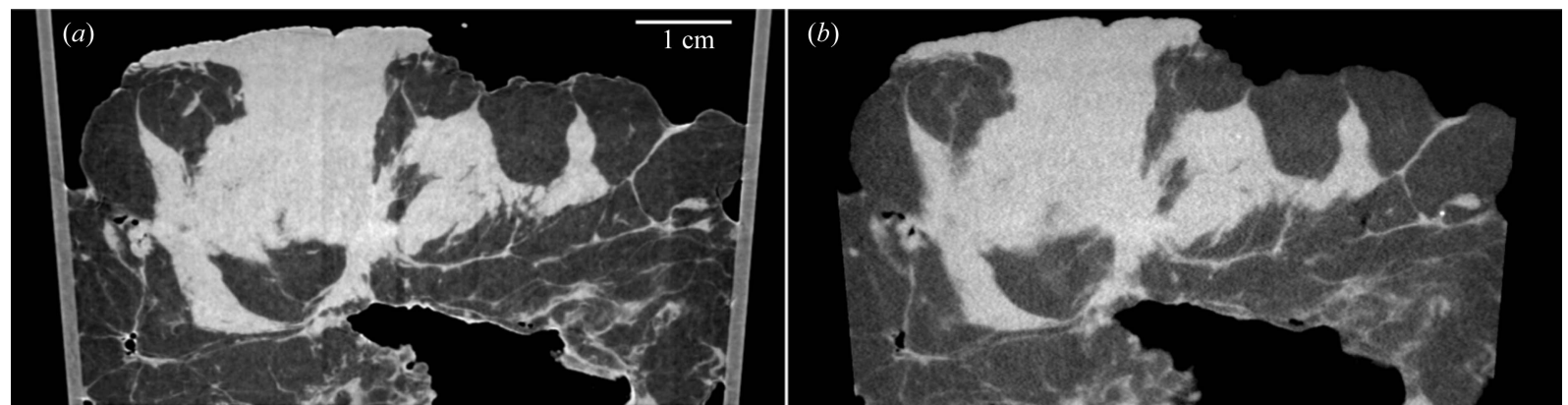

Figure 7

Example of a comparison between $(a)$ a PB-CT image and $(b)$ the reference image. The visualized plane is the sagittal one that corresponds to the mediolateral view of the two-dimensional mammography; the skin and the nipple are in the upper part.

coefficient (ICC) was calculated to obtain the inter-rater reliability (Koo \& Li, 2016). The ICC for the overall quality (average of all quality criteria for each image) was 0.924 , which indicates moderate reliability in the assessment. Visual grading analysis (VGA) was then conducted and the relative VGA score $\left(\mathrm{VGAS}_{\mathrm{rel}}\right)$ of the phase-contrast images was calculated using the following formula,

$$
\operatorname{VGAS}_{\mathrm{rel}}=\frac{\sum_{1}^{I} \sum_{1}^{C} \sum_{1}^{A} G_{i, c, a}}{I C A},
$$

where $G_{i, c, a}$ is the relative rating for a particular image $(i)$, criterion (c) and assessor $(a) . I, C$ and $A$ are the number of images, criteria and assessors, respectively (Sund et al., 2004). Fig. 7 presents an example of a comparison between the reference image and a PB-CT image, and the improvement in image quality brought about by the phase retrieval is clearly noticeable.

The VGAS $\mathrm{V}_{\text {rel }}$ of phase-contrast CT images was found to be 0.99 in this study, meaning that, on average, they were of higher quality than conventional absorption CT images. Note that a positive outcome of a comparison between two types of image (indicating higher quality of the test images than the reference) could have any value of $\mathrm{VGAS}_{\text {rel }}$ higher than 0 and lower than or equal to 2 , negative results correspond to VGAS $_{\text {rel }}$ values between -2 (inclusive) and 0 (exclusive), and the equivalence of two types of image corresponds to a VGAS $_{\text {rel }}$ value of 0.

\section{Conclusions}

We have been able to demonstrate that propagation phasecontrast $\mathrm{CT}$ applied to imaging a fresh breast-tissue specimen shows the same improved image quality as was previously found in several studies using formalin-fixed breast-tissue sections. This paves the way for a future application for breast imaging of live patients. The PB-CT case presented here has clearly demonstrated an increased diagnostic power compared with conventional medical imaging methods. We have found that, by using phase-contrast breast CT as a diagnostic tool, a more precise characterization of the lesion can be achieved, leading to a more accurate diagnosis. Moreover, the results from the visual grading analysis study undertaken by expert radiological assessors convincingly showed PB-CT imaging using synchrotron radiation can lead to an improvement in radiological image quality while maintaining an X-ray dose at a clinically acceptable level.

\section{Funding information}

The following funding is acknowledged: Australian National Breast Cancer Foundation (grant No. IN-16-001).

\section{References}

Alakhras, M., Bourne, R., Rickard, M., Ng, K. H., Pietrzyk, M. \& Brennan, P. C. (2013). Clin. Radiol. 68, e225-e236.

Baran, P. et al. (2017). Phys. Med. Biol. 62, 2315-2332.

Bravin, A., Coan, P. \& Suortti, P. (2013). Phys. Med. Biol. 58, R1-R35.

Ciatto, S., Houssami, N., Bernardi, D., Caumo, F., Pellegrini, M., Brunelli, S., Tuttobene, P., Bricolo, P., Fantò, C., Valentini, M., Montemezzi, S. \& Macaskill, P. (2013). Lancet Oncol. 14, 583-589.

Coan, P., Bravin, A. \& Tromba, G. (2013). J. Phys. D Appl. Phys. 46, 494007.

Diemoz, P. C., Bravin, A. \& Coan, P. (2012). Opt. Express, 20, 2789 2805.

Elmore, J. G., Armstrong, K., Lehman, C. D. \& Fletcher, S. W. (2005). JAMA, 293, 1245-1256.

Gureyev, T. E., Nesterets, Y., Ternovski, D., Thompson, D., Wilkins, S. W., Stevenson, A. W., Sakellariou, A. \& Taylor, J. A. (2011). Proc. SPIE, 8141, 81410B.

Heywang-Köbrunner, S. H., Hacker, A. \& Sedlacek, S. (2011). Breast Care (Basel), 6, 199-207.

Joy, J. E., Penhoet, E. E. \& Petitti, D. B. (2005). Editors. Benefits and Limitations of Mammography. Washington DC: US National Academies Press.

Keyriläinen, J., Fernández, M., Bravin, A., Karjalainen-Lindsberg, M.-L., Leidenius, M., von Smitten, K., Tenhunen, M., Kangasmäki, A., Sipilä, P., Nemoz, C., Virkkunen, P. \& Suortti, P. (2011). J. Synchrotron Rad. 18, 689-696.

Kidane, G., Speller, R. D., Royle, G. J. \& Hanby, A. M. (1999). Phys. Med. Biol. 44, 1791-1802.

Koo, T. K. \& Li, M. Y. (2016). J. Chiropr. Med. 15, 155-163.

Longo, R. et al. (2016). Phys. Med. Biol. 61, 1634-1649.

Michell, M. J., Iqbal, A., Wasan, R. K., Evans, D. R., Peacock, C., Lawinski, C. P., Douiri, A., Wilson, R. \& Whelehan, P. (2012). Clin. Radiol. 67, 976-981.

Nesterets, Y. I., Gureyev, T. E., Mayo, S. C., Stevenson, A. W., Thompson, D., Brown, J. M. C., Kitchen, M. J., Pavlov, K. M., Lockie, D., Brun, F. \& Tromba, G. (2015). J. Synchrotron Rad. 22, 1509-1523.

Pacilè, S., Brun, F., Dullin, C., Nesterets, Y. I., Dreossi, D., Mohammadi, S., Tonutti, M., Stacul, F., Lockie, D., Zanconati, F., 
Accardo, A., Tromba, G. \& Gureyev, T. E. (2015). Biomed. Opt. Expr. 6, 3099-3112.

Paganin, D. (2013). Coherent X-ray Optics. Oxford University Press.

Paganin, D., Mayo, S. C., Gureyev, T. E., Miller, P. R. \& Wilkins, S. W. (2002). J. Microsc. 206, 33-40.

Pagot, E., Fiedler, S., Cloetens, P., Bravin, A., Coan, P., Fezzaa, K., Baruchel, J., Härtwig, J., von Smitten, K., Leidenius, M., Karjalainen-Lindsberg, M. L. \& Keyriläinen, J. (2005). Phys. Med. Biol. 50, 709-724.

Rigon, L. (2014). Comprehensive Biomedical Physics, edited by A. Brahme, pp. 193-220. Oxford: Elsevier.

Siegel, R. L., Miller, K. D. \& Jemal, A. (2018). CA Cancer J. Clin. 68, 7-30.

Sprague, B. L., Arao, R. F., Miglioretti, D. L., Henderson, L. M., Buist, D. S. M., Onega, T., Rauscher, G. H., Lee, J. M., Tosteson, A. N. A.,
Kerlikowske, K., Lehman, C. D. \& Breast Cancer Surveillance Consortium (2017). Radiology, 283, 59-69.

Sund, P., Båth, M., Kheddache, S. \& Månsson, L. G. (2004). Eur. Radiol. 14, 48-58.

Sztrókay, A., Diemoz, P. C., Schlossbauer, T., Brun, E., Bamberg, F., Mayr, D., Reiser, M. F., Bravin, A. \& Coan, P. (2012). Phys. Med. Biol. 57, 2931-2942.

Tromba, G., Pacilè S., Nesterets, Y. I., Brun, F., Dullin, C., Dreossi, D., Mayo, S. C., Stevenson, A. W., Pavlov, K. M., Kitchen, M. J., Thompson, D., Brown, J. M. C., Lockie, D., Tonutti, M., Stacul, F., Zanconati, F., Accardo, A. \& Gureyev, T. E. (2016). Lect. Notes Comput. Sci. 9699, 625-634.

Zhao, Y., Brun, E., Coan, P., Huang, Z., Sztrókay, A., Diemoz, P. C., Liebhardt, S., Mittone, A., Gasilov, S., Miao, J. \& Bravin, A. (2012). Proc. Natl Acad. Sci. USA, 109, 18290-18294. 